\title{
Primary Dysmenorrhea and Menstrual Symptoms in Indian Female Students: Prevalence, Impact and Management
}

\author{
Shabnam Omidvar ${ }^{1}$, Fatemeh Bakouei ${ }^{1}$, Fatemeh Nasiri Amiri ${ }^{1} \&$ Khyrunnisa Begum $^{2}$ \\ ${ }^{1}$ Department of Midwifery, Babol University of Medical Sciences Babol, Iran \\ ${ }^{2}$ Department of Studies in Food Science and Nutrition, University of Mysore, Mysore, India \\ Correspondence: Fatemeh Bakouei, Department of Midwifery, Babol University of Medical Sciences Babol, Iran. \\ Tel: 98-911-315-8440. E-mail: bakouei2004@yahoo.com
}

\author{
Received: September 30, 2015 Accepted: November 24, 2015 Online Published: December 17, 2015 \\ doi:10.5539/gjhs.v8n8p135 \\ URL: http://dx.doi.org/10.5539/gjhs.v8n8p135
}

\begin{abstract}
Background: Dysmenorrhea is the most common gynecological problem among females and it is defined as cramping pain in the lower abdomen occurring just before or during menstruation. Menstrual symptoms are a broad collection of affective and somatic concerns that occur around the time of menses. The effect and importance of dysmenorrhea is very wide, therefore managing the problem is important.
\end{abstract}

Objective: To ascertain the prevalence, and impact of primary dysmenorrhea in student girls and their management behaviors.

Methods and Materials: A Cross-sectional study was conducted on 1000 healthy females aged 11-28 years. Standardized Self-reporting questionnaires were used to obtain relevant data. Pain intensity was assessed by using the Numerical Pain Scale (NPS). Data was analyzed by SPSS version 16.

Results: Prevalence of dysmenorrhea was $70.2 \%$. Majority of the subjects experienced pain for one or 1-2 days during menstruation. $23.2 \%$ of the dysmenorrheic girls experienced pain for 2-3 days. The most common symptom in both dysmenorrheic and non dysmenorrheic girls during the menstrual periods was tiredness and second most prevalent symptom was back pain.

Females experiencing mild pain on an average absented for one and half day a month while 2.1 \pm 1.2 and $2.5 \pm 1.3$ days for those who experienced moderate and severe forms of dysmenorrhea respectively. A small proportion of girls sought pharmacological management (25.5\%) and 83.2\% depended on non-pharmacological methods. Only $14.2 \%$ had sought medical advice.

Conclusion: Sub optimal use of the medical advice and the barriers to seek medical attention by dysmenorrheic females need exploration. It is important that health education on puberty and menstruation is regarded as inadequate for many girls in India.

Keywords: primary dysmenorrhea, impact, management, menstrual symptoms

\section{Introduction}

Dysmenorrhea is a cyclical lower abdominal or pelvic pain which may also radiate to the back and thighs; it occurs before or during menstruation, or both (Raine-Fenning, 2005). Cramps and pain are experienced in the lower abdominal after regular ovulation is established. It begins soon after menarche (Reddish, 2006). It is the most common gynecologic complaint among adolescent and adult females (Doty \& Attaran, 2006; Polat, Celik et al., 2009).

Menstruation-associated symptoms are a broad collection of affective and somatic concerns that occur around the time of menses (Harel, 2006; Negriff, 2009). Symptoms, such as headache, vomiting, tiredness, dizziness, and diarrhea are few commonly experienced menstrual symptoms. Symptoms typically start at the onset of menstrual flow or occur within a few hours before or after onset, and last for the first 24-48 hours.

The epidemiology of primary dysmenorrhea is difficult to establish since it is a symptom that is perceived differently by different women and also diversity in diagnostic criteria that is often used. The prevalence estimates range from 25 to 90\% among women and adolescents (Okusanya, 2009). Studies from India reported the prevalence range between 50 to $87.8 \%$ (George \& Bhaduri, 2002; Patel, 2006; Nair, 2007; Singh, 2008; 
Agarwal \& Agarwal, 2010). Other studies reported that dysmenorrhea affects up to $90 \%$ of women of childbearing age to varying degrees (Weissman, 2004). Pain is significant in $5-20 \%$ who report severe dysmenorrhea or pain that prevents them from participating in their usual activities. In the United States, the annual economic loss has been estimated as 600 million work hours worth 2 billion dollars. The effect and importance of dysmenorrhea is very wide and not restricted to its socio-economic impact (Raine-Fenning, 2005).

Although etiology of dysmenorrhea is not fully understood but the cause of pain at, or around, the time of menses is believed to be due to the production of prostaglandins in the endometrium in an ovulatory cycle. There are some reports that indicate the levels of prostaglandin F2 $\alpha$ measured in menstrual fluid from tampons and found to be twice higher in the dysmenorrheic as against the non dysmenorrheic women (Harel, 2006). As a remedial measure significant beneficial effect of prostaglandin inhibitors is reported in patients with dysmenorrhea. Despite the evidence supporting a link between prostaglandin levels and dysmenorrhea, it is important to recognize that the explanation for menstrual pain is not as simplistic as the cyclic production of one hormone. Women with dysmenorrhea may have complex alterations in hormonal patterns that exist throughout the cycle (Sharp, 2002). Treatment for dysmenorrhea is aimed at relieving symptoms and is best treated with analgesics that are prostaglandin inhibitors (Shilpa, 2008). The objective of this study was to ascertain the prevalence of dysmenorrhea and menstrual symptoms in Indian girls, its impact on their performance and treatment approaches.

\section{Methods and Materials}

The present study was of a Cross-sectional design conducted in an urban area in a major city in South India. The selected subjects were explained about the protocol and purpose of the study and were requested to complete the questionnaires to gather information relating to demographic feature, menstrual pain, intensity of pain, pain characteristics, menstrual symptoms, impact of dysmenorrhea and symptoms on their daily chores and their menstrual pain management methods. One thousand healthy young females aged 11-28 years formed the study population. A purposeful sampling was adopted to select unmarried girls; also those who volunteered to give complete and correct information were included for the study. The demographic information included family details relating to family size and type, chronological age and age at menarche. Possession of costly goods like vehicles, computer, TV, DVD, refrigerator, phones was also obtained and this information was used to compute Socio Economic Status (SES); it was designated as low, middle and high SES. The Numerical Pain Scale developed by McCaffery and Beebe (1994) was used for assessing pain intensity (McMaffery, 1994). The research protocol was approved by the Ethical Committee, University of Mysore.

The data was analyzed using SPSS for Windows version 16. Descriptive statistics was used to determine mean and percentages. The categorical data was analyzed using Chi-sq, correlation and regression analyses.

\section{Results}

Table 1 provides participants general and family information. The proportion of participants belonging to the four age categories $(11-15,16-18,19-21,>22$ years) were essentially similar. It is evident that majority of the selected girls belonged to Hindu families, while those from Islam, Christianity and other religions varied from $1.3-12.3 \%$. Nearly half of the subjects had completed or presently studying in $10^{\text {th }}$ standard or lower than that, 33.2 and $19.4 \%$ were studying in pre university and graduate classes. It can be seen that regardless of the SES; the predominant family type was nuclear family with members $\leq 4$. Joint family and the extended families together formed $14 \%$ wherein the size of the family varied from 5-8 members. Large families with more than 8 members were insignificant. More than half of the subjects belonged to medium SES followed by low SES (36.7\%). It was surprising that a considerable percentage of the selected subjects mentioned to have a positive family history of menstrual pain.

Table1. Demographic information about the participants

\begin{tabular}{llll}
\hline Information & & $\mathbf{N}$ & $\mathbf{\%}$ \\
\hline \multirow{3}{*}{ Age } & $11-15$ & 226 & 22.6 \\
(years) & $16-18$ & 252 & 25.2 \\
& $19-21$ & 271 & 27.1 \\
\multirow{2}{*}{ Religion } & $\geq 22$ & 251 & 25.1 \\
& Hindu & 836 & 84.0 \\
& Muslim & 123 & 12.3 \\
\hline
\end{tabular}




\begin{tabular}{llll}
\hline Information & & $\mathbf{N}$ & $\mathbf{\%}$ \\
\hline & Christian & 24 & 2.4 \\
& Other & 13 & 1.3 \\
Education & Up to $10^{\text {th }}$ std & 469 & 47.0 \\
& Pre university & 332 & 33.2 \\
& Graduation & 194 & 19.4 \\
Type of family & Post graduation & 4 & 0.4 \\
& Joint & 64 & 6.4 \\
& Nuclear & 852 & 85.7 \\
Family size & Extended & 78 & 7.8 \\
& $\leq 4$ & 538 & 53.9 \\
SES & $5-8$ & 442 & 44.3 \\
& $>8$ & 18 & 1.8 \\
Family history in menstrual pain & Low & 367 & 36.7 \\
& Medium & 529 & 52.9 \\
& High & 104 & 10.4 \\
& Yes & 622 & 64.5 \\
& No & 342 & 35.5 \\
\hline
\end{tabular}

$70.2 \%$ of the participants experienced menstrual pain, i.e. they were dysmenorrheic (Table 2). It was a point of concern since it is an indication of discomfort and poor work efficiency of the person. Length of pain varied for one day to the entire period of menstruation. Majority of them experienced pain for one or 1-2 days during menstruation. Furthermore, it is important to mention that $23.2 \%$ of the participants experienced pain for $2-3$ days and a small percent had for the entire period.

It was of interest to investigate the frequency of occurrence of dysmenorrhea. Intra and inter individual variations have been reported in the frequency of dysmenorrhea. A variety of factors are indicated to influence dysmenorrheal, most importantly are the psychological factors like stress, nutritional factors and hormonal effects. In the study population, girls with dysmenorrhea, (68.3\%) mentioned consistent pain during periods. On the other hand 20 and $11.8 \%$ dysmenorrheic girls reported having pain; less frequently and rarely respectively (Table 2). Approximately $50 \%$ of the girls in the study group remained absent from schools or colleges due to pain and/or discomfort related to menstruation. Only $25.6 \%$ of the dysmenorrheic participants mentioned to manage pain by taking analgesic Tablets.

Table 2. Dysmenorrhea and associated features: occurrence of dysmenorrhea among the selected females

\begin{tabular}{llll}
\hline Dysmenorrhea variables & & N & $\mathbf{( \% )}$ \\
\hline Menstrual pain & Yes & 698 & 70.2 \\
& No & 296 & 29.8 \\
Pain length & One day & 213 & 30.1 \\
& $1-2$ days & 288 & 40.7 \\
& $2-3$ days & 164 & 23.2 \\
Pain intensity & Entire of period & 42 & 6 \\
& Mil & 211 & 30.2 \\
Menstrual symptoms & Moderate & 333 & 47.7 \\
& Severe & 154 & 22.1 \\
Frequency of dysmenorrhea occurrence & Yes & 909 & 91.3 \\
& No & 87 & 8.7 \\
& More frequently (every month) & 458 & 68.3 \\
& Less frequently & 134 & 20.0 \\
\hline
\end{tabular}




\begin{tabular}{lllll}
\hline Dysmenorrhea variables & & & N & (\%) \\
\hline & Rarely(Once in 6 months) & 79 & 11.8 \\
& No & & 349 & 49.9 \\
& & Half day & 97 & 13.9 \\
Absenteeism because of menstrual pain/symptoms & Yes & 1 day & 173 & 24.8 \\
& & 2 days & 44 & 6.3 \\
& & $\geq 3$ days & 36 & 5.2 \\
Taking analgesics & Yes & & 178 & 25.6 \\
& No & & 517 & 74.4 \\
\hline
\end{tabular}

Our results exhibited that $69.7 \%$ of subjects were moderately affected, limiting their working capacity and $7.6 \%$ of both dysmenorrheic and non dysmenorrheic mentioned to have a clear inhibition of their activities.

Table 3. Ability of the participants to attend daily chores during menstrual time: differences between dysmenorrheic and non dysmenorrheic females

\begin{tabular}{lllll}
\hline Attending daily chores & Dysmenorrheic & Non dysmenorrheic & Total & Chi Square \\
\hline Unaffected & $\mathbf{1 5 5}(\mathbf{2 2 . 7})$ & $\mathbf{1 3 4}(\mathbf{4 8 . 2})$ & $\mathbf{2 8 9}$ & $\mathbf{6 3 . 5 1 6}$ \\
Moderately affected & $\mathbf{4 7 7}(69.7)$ & $\mathbf{1 2 3}(\mathbf{4 4 . 2})$ & $\mathbf{6 0 0}$ & \\
Clearly inhibited & $52(7.6)$ & $21(7.6)$ & 73 & \\
\hline
\end{tabular}

Table 4 presents association between certain subjective factors with intensity of menstrual pain. It is obvious that age and SES did not have any association with pain intensity. Religion exhibited small but significant association to pain; wherein higher percentage of girls from Hindu religion were found to have moderate pain followed by Christians while higher percentage of Muslim girls experienced severe pain.

Exercise as a purposive activity was seen in a small percentage of the study population (12.7\%). An essentially similar percentage of participants from varying pain intensity performed regular exercise therefore, statistical significance was not found. Among the other factors, association of pain intensity with anemia and absenteeism was highly significant $(\mathrm{P}=0.000)$. Absenteeism was more common in those with increasing severity of dysmenorrhea. The mean number of days of absence from schools and colleges for those with moderate to severe dysmenorrhea was $2.21 \pm 1.2$ days (not given in the table). An attempt was made to identify the mean work days lost per person experiencing dysmenorrhea with varying intensities. Absenteeism was found linearly associated to the severity of dysmenorrhea. Females experiencing mild pain on an average absented for one and half day a month while $2.1 \pm 1.2$ and $2.5 \pm 1.3$ days for those who experienced moderate and severe forms of dysmenorrhea respectively. However, the absolute number of days of absenteeism ranged from half a day to 3 days. Evidently the panorama of dysmenorrhea with its menstrual characteristics affects working ability of the females. An extremely significant association was found between the severity of dysmenorrhea and limitation in working capacity of females. An inverse relationship is exhibited between severity of dysmenorrhea and percentage of females reporting unaffected working ability while a proportional increase can be seen in percentage of females reporting clear inhibition of working ability.

Pain characteristics were also investigated in dysmenorrheic females. Location of pain is considered as an important criterion. Pain characteristics show some association with pain intensity in the selected subjects. It is evident from Table 5 there was an association between pain location and severity of pain. The Chi-square test shows extremely significant associations between intensity of pain and frequency of occurrence of pain as well as the pain length. $(\mathrm{P}=0.000)$. It can be seen from Table 5 that abdominal pain was most frequent symptom in females experiencing dysmenorrhea regardless of its intensity. Other locations for pain reported by the participants were either sides of abdomen, low back pain and pain in more than one region. Our results point out to certain pattern of pain occurrence, the percentage of females with mild and moderate dysmenorrhea reported higher frequency for lower abdomen and low back pain as against those with severe dysmenorrhea. Pain in more than one location was reported by women with severe dysmenorrhea in significantly higher percentage. It could be possible that in severe dysmenorrhea the pain intensity being high a perception of defused pain in the entire region of abdomen and back may occur. 
Table 4. Association between subjective factors and pain intensity among dysmenorrheic girls

\begin{tabular}{|c|c|c|c|c|c|c|}
\hline \multirow{2}{*}{ Variables } & & \multirow[b]{2}{*}{$\mathbf{N}(\%)$} & \multicolumn{3}{|c|}{ Menstrual pain intensity } & \multirow[b]{2}{*}{ Chi sq } \\
\hline & & & $\begin{array}{l}\text { Mild n } \\
\text { (\%) }\end{array}$ & $\begin{array}{l}\text { Moderate n } \\
\text { (\%) }\end{array}$ & $\begin{array}{l}\text { Severe n } \\
(\%)\end{array}$ & \\
\hline \multirow{4}{*}{ Age (Yrs) } & $12-15$ & $156(22.3)$ & $48(22.7)$ & $72(21.6)$ & $36(23.4)$ & \multirow{4}{*}{$4.103^{\mathrm{Ns}}$} \\
\hline & $16-18$ & $159(22.8)$ & $42(19.9)$ & $84(25.2)$ & $33(21.4)$ & \\
\hline & $19-21$ & $203(29.1)$ & $70(33.2)$ & $89(26.7)$ & $44(28.6)$ & \\
\hline & $>21$ & $180(25.8)$ & $51(24.2)$ & $88(26.4)$ & $41(26.6)$ & \\
\hline \multirow{3}{*}{$\begin{array}{l}\text { Menarche age } \\
\text { (Yrs) }\end{array}$} & $10-12$ & 191 & $52(25.7)$ & $93(28.7)$ & $46(30.5)$ & \multirow{3}{*}{$1.95^{\mathrm{Ns}}$} \\
\hline & $13-14$ & 387 & $117(57.9)$ & $183(56.5)$ & $87(57.6)$ & \\
\hline & $15-17$ & 99 & $33(16.3)$ & $48(14.8)$ & 18(11.9) & \\
\hline \multirow{3}{*}{ SES } & Low & 261(37.4) & $81(38.4)$ & $127(38.1)$ & $53(34.4)$ & \multirow{3}{*}{$0.804^{\mathrm{Ns}}$} \\
\hline & Middle & $357(51.1)$ & $107(50.7)$ & $168(50.5)$ & $82(53.2)$ & \\
\hline & High & $80(11.5)$ & $23(10.9)$ & $38(11.4)$ & $19(12.3)$ & \\
\hline \multirow{3}{*}{$\begin{array}{l}\text { Anemia* (before } \\
6 \text { months) }\end{array}$} & Yes & $116(16.8)$ & $27(12.9)$ & $38(11.5)$ & $51(33.8)$ & \multirow{2}{*}{$40.08^{* * *}$} \\
\hline & No & $575(83.2)$ & $183(87.1)$ & $292(88.5)$ & $100(66.2)$ & \\
\hline & Yes & $88(12.7)$ & $33(15.7)$ & $33(10.0)$ & $22(14.3)$ & \\
\hline \multirow[t]{2}{*}{ Exercise } & No & $480(69.3)$ & $137(65.2)$ & $233(70.8)$ & $110(71.4)$ & \multirow[t]{2}{*}{$5.91^{\mathrm{Ns}}$} \\
\hline & sometimes & $125(18.0)$ & $40(19)$ & $63(19.1)$ & $22(14.3)$ & \\
\hline \multirow{5}{*}{ Absenteeism } & None & $330(49.3)$ & $128(67.0)$ & $151(46.3)$ & $51(33.6)$ & \multirow{5}{*}{$67.03^{* * *}$} \\
\hline & Half day & $90(13.5)$ & $30(15.7)$ & $45(13.8)$ & $15(9.9)$ & \\
\hline & 1 day & $170(25.4)$ & $25(13.0)$ & $90(27.6)$ & $55(36.2)$ & \\
\hline & 2 days & $44(6.6)$ & $5(2.6)$ & $25(7.7)$ & $14(9.2)$ & \\
\hline & 3 days & $35(5.2)$ & $3(1.6)$ & $15(4.6)$ & $17(11.2)$ & \\
\hline \multirow{3}{*}{ Working ability* } & Unaffected & 155 & $75(36.4)$ & $65(19.8)$ & $15(10.0)$ & \multirow{3}{*}{$49.28^{* * *}$} \\
\hline & $\begin{array}{l}\text { Moderately } \\
\text { affected }\end{array}$ & 477 & $126(61.2)$ & $238(72.6)$ & $113(75.3)$ & \\
\hline & Clearly inhibited & 52 & $5(2.4)$ & $25(7.6)$ & $22(14.7)$ & \\
\hline \multicolumn{2}{|c|}{ Percentage of absenteeism } & & 33.0 & 53.7 & 66.4 & \\
\hline \multicolumn{2}{|c|}{ Mean absent days } & & $1.6 \pm 0.9$ & $2.1 \pm 1.2$ & $2.5 \pm 1.3$ & \\
\hline
\end{tabular}

The descriptive terms used to characterize pain were twisting, sharp and stinging. All the dysmenorrheic females regardless of the severity reported essentially similar pain characteristics. The pattern of occurrence of pain was evident since a majority of females reported to experience twisting pain followed by sharp pain. Other information such as first experience of menstrual pain, frequency of pain occurrence, initiation and length of pain is also presented in the table. Considering the highest level of occurrence among the pain characteristics such as" experience of pain from the first period", "occurrence of pain every period", "initiation of pain from previous or first day" or" pain length" as the severe condition, it is obvious that severity of each variable mentioned, appeared with females having severe dysmenorrhea. Significantly higher percentage of girls with severe dysmenorrhea experienced pain every cycle, initiation of pain was reported from the first day and pain length was also long. This is a clear evidence of the sufferings among dysmenorrheic females which is directly proportional with mild, moderate and severe dysmenorrhea. We found extremely significant association between frequency of pain occurrence and dysmenorrhea as well as pain length and dysmenorrhea. 
Table 5. Distribution of pain characteristics according to dysmenorrhea intensity

\begin{tabular}{|c|c|c|c|c|c|c|}
\hline \multirow{2}{*}{ Pain characteristics } & & \multicolumn{3}{|c|}{ Menstrual pain intensity } & \multirow[b]{2}{*}{ Total } & \multirow[b]{2}{*}{ Chi Sq } \\
\hline & & $\begin{array}{l}\text { Mild n } \\
\text { (\%) }\end{array}$ & $\begin{array}{l}\text { Moderate } \\
\text { n (\%) }\end{array}$ & $\begin{array}{l}\text { Severe n } \\
(\%)\end{array}$ & & \\
\hline \multirow{4}{*}{ Pain Location } & Lower abdomen & $83(50.3)$ & $159(53.4)$ & $70(46.4)$ & $312(50.8)$ & \multirow{4}{*}{$25.3^{* *}$} \\
\hline & Low back & $46(27.9)$ & $48(16.1)$ & 19(12.6) & $113(18.4)$ & \\
\hline & sides of abdomen & $12(7.2)$ & $21(7.0)$ & $14(9.3)$ & $47(7.7)$ & \\
\hline & Pain at different locations & $24(14.6)$ & $70(23.5)$ & $48(31.8)$ & $142(23.1)$ & \\
\hline \multirow{4}{*}{ Pain Characters } & Twisting & $77(70.0)$ & $139(62.6)$ & $71(57.7)$ & $287(63.1)$ & \multirow{4}{*}{$9.36^{\mathrm{Ns}}$} \\
\hline & Sharp & $20(18.2)$ & $43(19.4)$ & $28(22.8)$ & $91(20.0)$ & \\
\hline & Stinging & $13(11.8)$ & $34(15.3)$ & $17(13.8)$ & $64(14.1)$ & \\
\hline & Mix & $0(0.0)$ & $6(2.7)$ & $7(5.7)$ & $13(2.8)$ & \\
\hline \multirow{3}{*}{$\begin{array}{l}\text { First experience of } \\
\text { menstrual pain }\end{array}$} & From the first period & $78(49.4)$ & $178(58.8)$ & $80(54.4)$ & $336(55.2)$ & \multirow{3}{*}{$4.03^{\mathrm{Ns}}$} \\
\hline & After one year & $46(29.1)$ & $68(22.4)$ & $36(24.5)$ & $150(24.7)$ & \\
\hline & Other & $34(21.5)$ & $57(18.8)$ & $31(21.1)$ & $122(20.1)$ & \\
\hline \multirow{3}{*}{$\begin{array}{l}\text { Frequency } \\
\text { occurrence pain }\end{array}$} & $\begin{array}{l}\text { More frequently (every } \\
\text { periods) }\end{array}$ & $90(44.5)$ & $237(74.3)$ & $131(87.3)$ & $458(68.2)$ & \multirow{3}{*}{$87.46^{* * *}$} \\
\hline & $\begin{array}{l}\text { Less frequently (Once in } 3 \\
\text { month) }\end{array}$ & $64(31.7)$ & $58(18.2)$ & $12(8.0)$ & $134(20.0)$ & \\
\hline & Rarely(Once in 6 months) & $48(23.8)$ & $24(7.5)$ & $7(4.7)$ & $79(11.8)$ & \\
\hline \multirow{5}{*}{ Initiation of pain } & Previous day & $47(23.6)$ & $76(23.2)$ & $36(23.7)$ & $159(23.4)$ & \multirow{5}{*}{$9.76^{\mathrm{Ns}}$} \\
\hline & First day & $98(49.2)$ & $180(55.0)$ & $86(56.6)$ & $364(53.7)$ & \\
\hline & Second day & $48(24.1)$ & $64(19.6)$ & $24(15.8)$ & $136(20.1)$ & \\
\hline & Later & $4(2.0)$ & $4(1.2)$ & $1(0.7)$ & $9(1.3)$ & \\
\hline & No fixed pattern & $2(1.0)$ & $3(0.9)$ & $5(3.3)$ & $10(1.5)$ & \\
\hline \multirow{4}{*}{ Pain length } & One day & $76(38.0)$ & $100(30.6)$ & $23(15.1)$ & $199(29.3)$ & \multirow{4}{*}{$27.44^{* * *}$} \\
\hline & $1-2$ days & $82(41.0)$ & $128(39.1)$ & $68(44.7)$ & $278(40.9)$ & \\
\hline & 2-3 days & $33(16.5)$ & $79(24.2)$ & $47(30.9)$ & $159(23.4)$ & \\
\hline & Entire of period & $9(4.5)$ & $20(6.1)$ & $14(9.2)$ & $43(6.4)$ & \\
\hline
\end{tabular}

The most common symptom present in both dysmenorrheic and non dysmenorrheic girls during the menstrual periods was tiredness (56.8 and $37.8 \%$ respectively) and second most prevalent symptom was back pain ( $40.1 \%$ and $17.6 \%)$.

The information provided in Table 6 is interesting since the differences in the occurrence rate of certain discomforts among dysmenorrheic and non dysmenorrheic females indicates the extent of sufferings the dysmenorrheic females undergo with each cycle of menstruation. Also, the table provides the common problems of females regardless of menstrual pain. The uterine contraction that occurs during menstruation is painful is generally accompanied with other symptoms, including tiredness, painful/tender breasts, supra pubic cramping, backache, general pain and vomiting.

Table 6. Distribution of the subjects according to the menstrual symptoms and occurrence of dysmenorrhea

\begin{tabular}{llll}
\hline \multirow{2}{*}{ Menstrual symptoms } & Groups & & \\
\cline { 2 - 4 } & Dysmenorrheic & Non dysmenorrheic & Chi sq \\
\hline Tiredness & $397(56.8)$ & $112(37.8)$ & $30.15^{* * *}$ \\
Back pain & $280(40.1)$ & $52(17.6)$ & $47.7^{* * *}$ \\
Anger/short tempered/irritable & $185(26.5)$ & $43(14.5)$ & $16.86^{* * *}$ \\
Mood swings & $122(17.5)$ & $28(9.5)$ & $10.43^{* *}$ \\
General aches and pains & $119(17.0)$ & $15(5.0)$ & $25.42^{* * *}$ \\
Acne & $105(15.0)$ & $34(11.5)$ & $2.13^{\mathrm{Ns}}$ \\
\hline
\end{tabular}




\begin{tabular}{llll}
\hline \multirow{2}{*}{ Menstrual symptoms } & Groups & & \\
\cline { 2 - 4 } & Dysmenorrheic & Non dysmenorrheic & Chi sq \\
\hline Headaches & $85(12.2)$ & $31(10.5)$ & $0.586^{\text {Ns }}$ \\
Insomnia & $63(9.0)$ & $10(3.4)$ & $9.74^{* *}$ \\
Vomiting & $57(8.1)$ & $7(2.4)$ & $11.61^{* * *}$ \\
Cramps & $56(8.0)$ & $2(0.6)$ & $20.42^{* * *}$ \\
Painful/tender breasts & $55(7.9)$ & $5(1.7)$ & $14.04^{* * *}$ \\
Altered appetite & $61(8.7)$ & $10(3.4)$ & $9.00^{* *}$ \\
Less frequent symptoms & & & \\
Nausea,diarrhea swelling, craving & $2.8-4.8$ & $0.6-3.4$ & \\
\hline
\end{tabular}

Management of pain adopted by the selected girls is also presented in Table 7. It is obvious that a small proportion of girls reported to have sought medical advice, nevertheless an insignificant percentage of girls used self selected medicine without doctor's consultation. Majority of dysmenorrheic females practiced staying in bed, having hot water bath, use of special food or drink to reduce pain and distraction by watching TV, reading etc. as a measure to relieve pain. As it can be seen from the table the most prevalent method was staying in bed.

Table 7. Menstrual pain management behaviors of the selected females

\begin{tabular}{|c|c|c|c|c|c|c|c|}
\hline \multirow{3}{*}{$\begin{array}{l}\text { Menstrual } \\
\text { pain intensity } \\
N=698\end{array}$} & \multicolumn{7}{|c|}{ Menstrual pain management (\%) } \\
\hline & \multicolumn{2}{|c|}{ Pharmacological supplements } & \multicolumn{5}{|c|}{ Non pharmacological Methods } \\
\hline & $\begin{array}{l}\text { over-the-counter } \\
\text { medication }\end{array}$ & $\begin{array}{l}\text { On doctors } \\
\text { consultation }\end{array}$ & Stay in bed & $\begin{array}{l}\text { Hot } \\
\text { bath/use of } \\
\text { heating pad }\end{array}$ & Distraction & $\begin{array}{l}\text { Special } \\
\text { food or } \\
\text { drink* }\end{array}$ & Exercise \\
\hline Mild & $15(19.7)$ & $31(17.4)$ & $121(25.4)$ & $63(28.5)$ & $45(26.0)$ & $32(30.2)$ & $9(39.1)$ \\
\hline Moderate & $36(47.4)$ & $74(41.6)$ & $236(49.6)$ & $107(48.4)$ & $84(48.6)$ & $47(44.3)$ & $6(26.1)$ \\
\hline Severe & $25(32.9)$ & $73(41.0)$ & $119(25.0)$ & $51(23.1)$ & $44(25.4)$ & $27(25.5)$ & $8(34.8)$ \\
\hline Total & $76(6.1)$ & $178(14.2)$ & $476(38.0)$ & $221(17.6)$ & $173(13.8)$ & $106(8.5)$ & $23(1.8)$ \\
\hline
\end{tabular}

*considered to relieve sufferings.

\section{Discussion}

We found $70.2 \%$ of females suffering from primary dysmenorrhea in selected population. Our observation is consistent with previous studies reporting dysmenorrhea occurrence between 45 and $85 \%$ from India and other countries (Loto, 2008; Okusanya, 2009; Agarwal \& Agarwal, 2010; Alaettin, 2010; Yasir, 2014). It is evident from results that nearly $70 \%$ of dysmenorrheic females experienced moderate to severe dysmenorrhea. While comparing prevalence of dysmenorrhea from other countries, Ethiopia reported $70 \%$ dysmenorrhea wherein $28.5 \%$ had moderate to severe, Malaysia reported an incidence of $6.8 \%$ having moderate to severe pain and Jordan reported $55.8 \%$ of the subjects had moderate to severe pain. One of the reasons for such vast differences could be due to the scales used for assessment (Lee, 2006; Zegeye, 2009; Al-Jefout, 2014).

In present study 20 and $11.8 \%$ dysmenorrheic girls reported having pain frequently and rarely (Table 2). The distribution pattern of pain frequency reported by the study population was found to be similar to the report from Hong Kong (Symphorosa, 2009). A study from India indicated 34\% females experience dysmenorrhea more frequently (every month) (Agarwal \& Agarwal, 2010). In comparison to this study, we found a markedly higher occurrence $(68.3 \%)$ of frequent dysmenorrhea. It is worthwhile to highlight that female with more frequent and less frequent experiences of dysmenorrhea was found to be $88 \%$ and this proportion is considerably higher.

We found approximately $50 \%$ of dysmenorrheic girls remained absent from schools or colleges. In several studies of young women, rates of absenteeism ranged from 24 to 50\% (Banikarim, 2000; Chia et al., 2013). Studies have demonstrated that menstrual pain restricts the movement and usual activity pattern of the females. It could also be argued that the perception of uneasiness and discomfort experienced during menstruation probably limits the movements more than the experience of pain. 
Results exhibited $69.7 \%$ of subjects were moderately affected and $7.6 \%$ of both dysmenorrheic and non dysmenorrheic mentioned to have a clear inhibition of their activities. Other studies also reported such activity restriction (Tariq, 2009; Al-Asadi, 2013).

Interestingly we found higher percentage of Muslim girls' experienced severe pain. A report has shown, that Muslim adolescents exhibited higher degrees of somatization, which may suggest that the cultural factor had a unique effect on the menstrual experiences (Goldestein-Ferber, 2006).

In only $12.7 \%$ of the study population exercise was a purposive activity. However, it is hypothesized that exercise works by improving blood flow at the pelvic level as well as stimulating the release of endorphins, which act as non-specific analgesics (Proctor \& Farquhar, 2006). A recent review showed that in studies where more than 500 participants were included there tended to be no association between dysmenorrhea and exercise/physical activity patterns but smaller studies ( $<500$ participants) reported positive associations, where bias is also more likely to be present (Daley, 2008).

We found tiredness and back pain as the most prevalent menstrual symptoms and our observations were similar to that reported by others (Agarwal \& Agarwal, 2010; Aktaş, 2015).

It is worthwhile to comment that, despite the sufferings, only a small proportion of girls in our study had sought pharmacological management $(25.5 \%)$ and $83.2 \%$ depended on non pharmacological methods. Only $14.2 \%$ had sought medical advice and this suboptimal use of the medical advice has also been reported by others (Banikarim, 2000; Lee, 2006; Konapur, 2014; Aktaş, 2015; Farotimi et al., 2015). The barriers to seek medical attention by dysmenorrheic females need exploration. It is important that health education on puberty and menstruation is regarded as inadequate for many young females in India and other nations. This ignorance might adversely affect the medical attention seeking behavior of dysmenorrheic females.

\section{Acknowledgements}

Authors are grateful to the all participants.

\section{Conflict of Interest}

The authors declare that there is no conflict of interests regarding the publication of this paper.

\section{References}

Agarwal, A. K., \& Agarwal, A. (2010). A study of dysmenorrhea during menstruation in adolescent girls. Indian Journal of Community Medicine: Official Publication of Indian Association of Preventive \& Social Medicine 35(1), 159-164. http://dx.doi.org/10.4103/0970-0218.62586

Aktaş, D. (2015). Prevalence and Factors Affecting Dysmenorrhea in Female University Students: Effect on General Comfort Level. Pain Management Nursing, 16(4), 534-543. http://dx.doi.org/10.1016/j.pmn.2014. 10.004

Al-Asadi Jn, A. Q. R. (2013). Dysmenorrhoea and its impact on daily routine activities among secondary school students in Basra, Iraq. J Fac Med Baghdad, 55, 339-344.

Al-Jefout, M. A. F., Hijazeen, S., Al-Qaisi, J., Al-Ma'aitah, R., Al-Ma'aitah, O., \& Luskomb, G. O. (2014). Dysmenorrhea: Prevalence \& Impact on Quality of Life among Young Adult Jordanian Females. Journal of Pediatric and Adolescent Gynecology.

Banikarim, C. C., \& Kelder, S. H. M. R. (2000). Prevalence and impact of dysmenorrhea on Hispanic female adolescents. Archives of Pediatrics and Adolescent Medicine, 154(12), 1226-1229. http://dx.doi.org/10. 1001/archpedi.154.12.1226

Chia, C., Lai, J. H., Cheung, P., Kwong, L., Lau, F. P., Leung, K., ..., Ngu, S. (2013). Dysmenorrhoea among Hong Kong university students: prevalence, impact, and management. Hong Kong Medical Journal. http://dx.doi.org/10.12809/hkmj133807

Daley, A. J. (2008). Exercise and primary dysmenorrhoea: A comprehensive and critical review of the literature. Sports Medicine, 38(8), 659-670. http://dx.doi.org/10.2165/00007256-200838080-00004

Doty, E., \& Attaran, M. (2006). Managing primary dysmenorrhea. Journal of pediatric and adolescent gynecology, 19(5), 341-344. http://dx.doi.org/10.1016/j.jpag.2006.06.005

Farotimi, A. A., Esike, J., Nwozichi, C. U., Ojediran, T. D., \& Ojewole, F. O. (2015). Knowledge, attitude, and healthcare-seeking behavior towards dysmenorrhea among female students of a private university in Ogun State, Nigeria. Journal of Basic and Clinical Reproductive Sciences, 4(1), 33-38. http://dx.doi.org/10. 


\section{3/2278-960X.153524}

George, A., \& Bhaduri, A. (2002). Dysmenorrhea among adolescent girls-symptoms experienced during menstruation. Health Promotion Educ, 17, 4.

Harel, Z. (2006). Dysmenorrhea in adolescents and young adults: Etiology and management. Journal of pediatric and adolescent gynecology, 19(6), 363-371. http://dx.doi.org/10.1016/j.jpag.2006.09.001

Ks Konapur, C. N. (2014). Dysmenorrhoea and Premensrtual Syndrome: Frequency and Effect on Daily Activities of Adolescent Girls in Rural Areas of Bangalore. International Journal of Medical Science and Public Health, 3(10), 1225-1228. http://dx.doi.org/10.5455/ijmsph.2014.090720143

Lee, L. C., Lee, P., \& Kk Kaur, J. (2006). Menstruation among adolescent girls in Malaysia: A cross-sectional school survey. Singapore Medical Journal, 47(10), 869-874.

Loto, O. M., Adewumi, T. A., \& Adewuya, A. O. (2008). Prevalence and correlates of dysmenorrhea among Nigerian college women. Australian and New Zealand Journal of Obstetrics and Gynaecology, 48(4), 442-444. http://dx.doi.org/10.1111/j.1479-828X.2008.00869.x

Mcmaffery, M. (1994). Pain: Clinical manual for nursing practice.

Nair, P. G. V., \& Kannan, A. (2007). Awareness and practices of menstruation and pubertal changes amongst unmarried female adolescents in a rural area of East Delhi. IJCM, 32(2), 156-157. http://dx.doi.org/10. 4103/0970-0218.35668

Negriff, S. D., Hillman, L. D., \& Huang, B. J. B. (2009). The measurement of menstrual symptoms. Journal of health psychology, 14(7), 899-908. http://dx.doi.org/10.1177/1359105309340995

Okusanya Bo, G. K., Gb, O., \& Ohiosimuan O. (2009). Menstrual pain and associated factors amongst undergraduates of Ambrose Alli University Ekpoma, Edo State, Nigeria. Nigerian Journal of Medicine: Journal of the National Association of Resident Doctors of Nigeria, 18(4), 409-412.

Patel, V., Tanksale, V., Sahasrabhojanee, M., Gupte, S., \& Nevrekar, P. (2006). The burden and determinants of dysmenorrhea: A population-based survey of 2262 women in Goa, India. BJOG, 113, 453-463. http://dx.doi.org/10.1111/j.1471-0528.2006.00874.X

Polat, A., Celik, H., Gurates, B., Kaya, D., Nalbant, M., Kavak, E., \& Hanay, F. (2009). Prevalence of primary dysmenorrhea in young adult female university students. Archives of gynecology and obstetrics, 279(4), 527-532. http://dx.doi.org/10.1007/s00404-008-0750-0

Proctor, M., \& Farquhar, C. (2006). Diagnosis and management of dysmenorrhoea. BMJ: British Medical Journal, 332(7550), 1134-1138. http://dx.doi.org/10.1136/bmj.332.7550.1134

Raine-Fenning, N. (2005). Dysmenorrhoea. Current Obstetrics \& Gynaecology, 15(6), 394-401. http://dx.doi. org/10.1016/j.curobgyn.2005.09.007

Reddish, S. (2006). Dysmenorrhoea. Australian family physician, 35(11), 842-849.

Sari Goldestein-Ferber, M. G. (2006). The Association between Somatization and Perceived Ability: Roles in Dysmenorrhea among Israeli Arab Adolescents. Psychosomatic Medicine, 68, 136-142. http://dx.doi.org/10. 1097/01.psy.0000197644.95292.00

Sharp Ba, T. D., Thomas, K., Killeen, M., \& Dawood, M. (2002). Cyclic perimenstrual pain and discomfort: The scientific basis for practice. J Obstet Gynecol Neonatal Nurs, 31, 637-649. http://dx.doi.org/10.1177/08 84217502239207

Shilpa Deb, N. R. F. (2008). Dysmenorrhea. Obstetrics, Gynecology and reproductive medicine, 18(11), $294-299$. http://dx.doi.org/10.1016/j.ogrm.2008.08.007

Singh, A. K., Singh, H. D., Nel, B., Singh, P., \& Tiwari, P. (2008). Prevalence and severity of dysmenorrhea: A problem related to menstruation, among first and second year female medical students. Indian J Physiol Pharmacol, 52(4), 389-397.

Symphorosa, S. C., Chan, K., Yuen, Y. P., Sahota, D., \& Chung, T. K. (2009). Menstrual problems and health-seeking behavior in Hong Kong Chinese girls Hong Kong. Med J, 15(1).

Tariq, N. H. M. et al. (2009). Impact and health care seeking behaviour of premenstrual symptoms and dysmenorrhoea. British Journal of Medical practioners, 2, 40-43.

Unsal, U. A. A., \& Tozun, M. (2010). A study of dysmenorrhea among female residents aged 18-45 years in 
semirural area of west turkey. Pak J Med Sci April-June, 26(2), 335-340.

Weissman, A. M. H., Hansen, M. D. A. J., \& Johnson, S. R. (2004). The natural history of primary dysmenorrhoea: A longitudinal study. BJOG: An International Journal of Obstetrics \& Gynaecology, 111(4), 345-352. http://dx.doi.org/10.1111/j.1471-0528.2004.00090.x

Women's Health Research Center News. (2003). University of Michigan Health System, 2, 6. University of Michigan Health Topics and the National Headache Foundation 2003. Retrieved February 4, 2004, from http://www2.med.umich.edu/prmc/media/relarch.cfm

Yasir, S., \& Dar Mf, K. B. (2014). Frequency of dysmenorrhoea, its impact and management strategies adopted by medical students. $J$ Ayub Med Coll Abbottabad, 26(3), 349-352.

Zegeye, B. M. A. A. M. D. T. (2009). Age at menarche and the menstrual pattern of secondary school adolescents in northwest Ethiopia. BMC Women's Health, 9(29). http://dx.doi.org/10.1186/1472-6874-9-29

\section{Copyrights}

Copyright for this article is retained by the author(s), with first publication rights granted to the journal.

This is an open-access article distributed under the terms and conditions of the Creative Commons Attribution license (http://creativecommons.org/licenses/by/3.0/). 